\title{
District Level Assessment of Spatial Clustering and Determinants of Diabetes Mellitus among Older Adolescents and Young Adults in India
}

\author{
Wahengbam Bigyananda Meitei ${ }^{1 *}$ \\ ${ }^{1}$ International Institute for Population Sciences, Mumbai, India \\ Corresponding Authors’ Email: *bigyanandawahengbam@gmail.com
}

\begin{abstract}
Globally non-communicable disease is the leading cause of death. Amongst all, diabetes mellitus (DM) represents the most prevalent non-communicable disease in India. However, the burden of DM shifting towards the lower ages has received relatively little attention in India. Thus, this study aims to assess the district level spatial clustering and determinants of DM among older adolescents and young adults. The study used data from the fourth round of the National Family Health Survey. A person in the age group 15-29 years of age is considered to be older adolescents and young adults. Moran's I and LISA were used to examine the presence of spatial autocorrelation and clustering respectively. Simultaneous Autoregressive Model was used for the assessment of determinants. Prevalence of DM across 640 districts ranged from 0.15 percent to 35 percent. Global Moran's I is 0.61 implying significant spatial correlation. There is significant high-high clustering of the prevalence of DM in the districts of northeastern states, eastern states, southern states and Delhi National Capital Regions. And, significant low-low clustering of the prevalence of DM in the districts of northern states and central India. Overweight and professionals who are physically inactive are at higher risk of acquiring DM. As the older adolescent and the young adults are the potential assets to the nation, reduction in the prevalence of DM among older adolescents and young adults is important and would be possible only if area-specific measures would be adopted on those clusters of districts where DM is high considering the other intervening covariates.
\end{abstract}

Keywords: Diabetes mellitus, spatial clustering, older adolescents, young adults

\section{Introduction}

Globally, non-communicable disease is the leading cause of death. Almost 67 percent of all deaths are due to non-communicable diseases (Mote 2016). Diabetes mellitus (DM), which is one of the major contributors to the burden of non-communicable diseases, is no longer the disease of opulent developed nations; its prevalence is increasing rapidly in developing countries (Mohan et al. 2017). In India, the contribution of DM to the burden of non-communicable diseases represents one of the most important among all other diseases. Every fifth diabetic case in the world is an Indian, and thus India is called the capital of diabetes (Joshi et al. 2007). According to the International Diabetes Federation South-East Asia, there were 69.1 million cases of DM in India in 2015 with a prevalence of 8.7 percent among the adults aged 20 to 79 years. The average cost of each of these patients is $\$ 94.9$. By 2040, the figure is expected to increase to 123.5 million cases (IDF 2015). The long-term economic implications of DM in developing countries are worrying (Bjork et al. 2003). The socioeconomic burden due to DM in India is one of the highest in the world (Bjork et al. 2003; Kapur 2007; Shashank et al. 2008). DM is a risk factor for various other diseases like cardiovascular, renal and atherosclerotic vascular disease (Koch et al. 1997; Sowers et al. 1988; Martin et al. 2014; 
Tuttolomonde et al. 2015). The rapid change in lifestyle associated with urbanization in combination with inevitable high genetic risks and behavioural risk factors are also a possible risk factor for the disease (Ramachandran et al. 1999; Tuomilehto et al. 2001; Whittemore et al. 2002; Simpson et al. 2003; Rejeski et al. 2012). Both in India and in the Western countries, obesity is one of the significant risk factors for DM (Chan et al. 1994; Steppan et al. 2001; Mohan et al. 2008). Due to urbanization, rise in living standards, increased sedentariness, and the cheap availability of calorie-rich fatty fast foods to all sections of people alike are also a few reasons for the worldwide increase in the prevalence of DM (Gupta et al. 2006; Diamond 2011).

The problem of DM is heterogeneous in India (Arora et al 2010; Anjana et al. 2017). Numerous studies have discussed the relationship between DM and its associated risk factors, but particularly for the adult and elderly population. However, the burden of DM shifting towards the lower ages has received relatively less attention in developing countries like India. And as the older adolescents and the young adults are the potential assets to the nation, this paper aims to highlight the clustering of prevalence and determinants of DM among the older adolescent and young adults in India.

\section{Methods and Materials}

\section{Data and Variables}

The district-level information for all 640 districts in India was obtained from the fourth round of the National Family Health Survey. The survey was conducted in 2015-16 across all districts of all states and union territories of India. The survey collects information on population, health, and nutrition for each state and union territory. Apart from these, the survey also collects information on clinical, anthropometric and biomedical data to provide vital information on prevalence of hypertension, blood glucose levels as well as some other nutritional indicators through a series of biomarkers, tests and measurements (IIPS and ICF 2017).

We used the blood glucose level provided in the survey to identify whether a person is diabetic or not. A person whose blood glucose level is greater than $140 \mathrm{mg} / \mathrm{dL}$ is considered to be diabetic. And, persons in the age group $15-29$ years of age are defined as older adolescents and young adults (Bleyer 2002).

The unit of analysis for the study is the districts of the states and union territories of India. The outcome variable is the prevalence of DM among older adolescents and young adults in each of the 640 districts. The choice of predictor variables are governed by the existing literature (Whittemore $e t$ al. 2002; Mokdad et al. 2003; Savoca et al. 2004; Pereira et al. 2005). The fourth round of the National Family Health Survey provides household socioeconomic characteristics and community characteristics such as wealth quintile and place of residence. The wealth quintile of a household is categorized as - poorest, poorer, middle, richer and richest. A person is categorized as professional who are physically inactive if he/she is in a managerial, clerical and sales professions. Persons whose body mass index is greater than 24.9 are categorized as overweight. The survey also provides behavioural risk factors of DM such as consumption of tobacco, smoking and drinking alcohol. Thus we used the district level percentage of urban household, percentage of household with richest wealth quintile, percentage of the overweight population, percentage of professionals who are physically inactive and the behavioural risk factors viz. prevalence of tobacco consumption, smoking and 
drinking alcohol as the predictors of the prevalence of DM. The information on the frequency of intake of nine food groups was also collected in the survey out of which four are vegetarian food items viz. (milk, pulses or beans, dark green leafy vegetables and fruits), and three are non-vegetarian food items viz. eggs, fish and meat and the remaining two are fried food and carbonated drinks. Thus, we also consider these food habit patterns of the households as the prognosticator of DM.

\section{Statistical Analysis}

Univariate exploratory spatial data analysis such as Moran's I, a univariate local indicator for spatial correlation (LISA) and simultaneous autoregressive model (SAR) was used to assess the spatial clustering and determinants of DM among older adolescents and young adults.

Moran's I, statistic is defined as,

$I=\frac{n}{S_{0}} \frac{\sum_{i} \sum_{j} w_{i j}\left(y_{i}-\bar{y}\right)\left(y_{j}-\bar{y}\right)}{\sum_{i}\left(y_{i}-\bar{y}\right)^{2}}$, Where $S_{0}=\sum_{i} \sum_{j} w_{i j}$

Here we assume that the global means $\bar{y}$ is an adequate representation of the variable of interest $\mathrm{y}$. Moran's I value ranged from -1 to +1 . Negative values indicate negative spatial autocorrelation and positive values indicate positive spatial autocorrelation. A zero value indicates a random spatial pattern.

The index which is used to observe spatial autocorrelation at the local level is LISA, which can be seen as the local equivalent of Moran's I. For each location, LISA values allow for the computation of its similarity with its neighbours and also test its significance. Thus, five scenarios may emerge (Gupta et.al. 2016): (a) Locations with high values, with similar neighbours (high-high), referred to as "hot spots", (b) Locations with low values, with similar neighbours (low-low), referred to as "cold spots", (c) Locations with high values, but with low-value neighbours (high-low), referred to as potential "spatial outliers", (d) Locations with low values, but with high-value neighbours (low-high), also referred to as potential "spatial outliers", (e) Locations with no significant local autocorrelation.

The Spatial Autoregressive Models (SAR) specification uses a regression on the value from the other areas to account for the spatial dependence (Robert Gentleman et.al. 2008). Which means that the error terms $\varepsilon$ are modelled so that they depend on each other in the following way,

$\mathrm{e}_{\mathrm{i}}=\sum_{\mathrm{i}=1}^{\mathrm{m}} \mathrm{b}_{\mathrm{ij}} \mathrm{e}_{\mathrm{i}}+\varepsilon_{\mathrm{i}}$

Where $\varepsilon_{\mathrm{i}}$ are residuals errors which are assumed to be independently distributed according to a

Normal distribution with mean zero and diagonal covariance matrix $\Sigma_{\varepsilon}$ with elements $\sigma_{\varepsilon}^{2}, \mathrm{i}=1, \ldots, \mathrm{m}$. 
The $b_{i j}$ values are used to represent spatial dependence between areas, $b_{\mathrm{ij}}$ must be set to zero so that each area is not regressed on itself.

If we express the error terms as, $\mathrm{e}=\mathrm{B}\left(\mathrm{Y}-\mathrm{X}^{\mathrm{T}} \beta\right)+\varepsilon$, the model can be expressed as

$\mathrm{Y}=\mathrm{X}^{\mathrm{T}} \beta+\mathrm{B}\left(\mathrm{Y}-\mathrm{X}^{\mathrm{T}} \beta\right)+\varepsilon$

Which implies that,

$(I-B)\left(\mathrm{Y}-\mathrm{X}^{\mathrm{T}} \beta\right)=\varepsilon$

Where $\mathrm{B}$ is a matrix that contains the dependence parameters $\mathrm{b}_{\mathrm{ij}}$ and $\mathrm{I}$ is the identity matrix. In order that the SAR model to be well defined, the matrix I - B must be non-singular. Under this model, Y is distributed according to multivariate normal mean, $E[Y]=X^{T} \beta$ and covariance matrix

$\operatorname{Var}[\mathrm{Y}]=(\mathrm{I}-\mathrm{B})^{-1} \Sigma_{\varepsilon}\left(\mathrm{I}-\mathrm{B}^{\mathrm{T}}\right)^{-1}$

Often $\Sigma_{\varepsilon}$ is taken to depend on a single parameter $\sigma^{2}$, so that ${ }^{\Sigma_{\varepsilon}}=\sigma^{2} \mathrm{I}$ and then $\operatorname{Var}[\mathrm{Y}]$ simplifies to

$\operatorname{Var}[\mathrm{Y}]=\sigma^{2}(\mathrm{I}-\mathrm{B})^{-1}\left(\mathrm{I}-\mathrm{B}^{\mathrm{T}}\right)^{-1}$

It is also possible to specify $\Sigma_{\varepsilon}$ as a diagonal matrix of weights associated with heterogeneity among the observations. If $B=\lambda W$, where $\lambda$ is a spatial autocorrelation parameter and $\mathrm{W}$ is a matrix that represents spatial dependence, which is often assumed to be symmetric.

\section{Results}

\section{Descriptive Findings}

Figure 1 presents the distribution of the prevalence of DM among older adolescents and young adults across 640 districts of India. Its prevalence varied widely ranging from 0.57 percent to 35 percent and it is spread across all the districts of the country. Some of the Indian districts have a prevalence of less than 5 percent while many of them have prevalence more than 10 percent. Compared to districts in northern states, districts in eastern, northeastern, western, and southern states seem to be better as almost all the districts have prevalence less than 5 percent. Many of the districts in northern, western, central and northeastern states have prevalence more than 10 percent. 


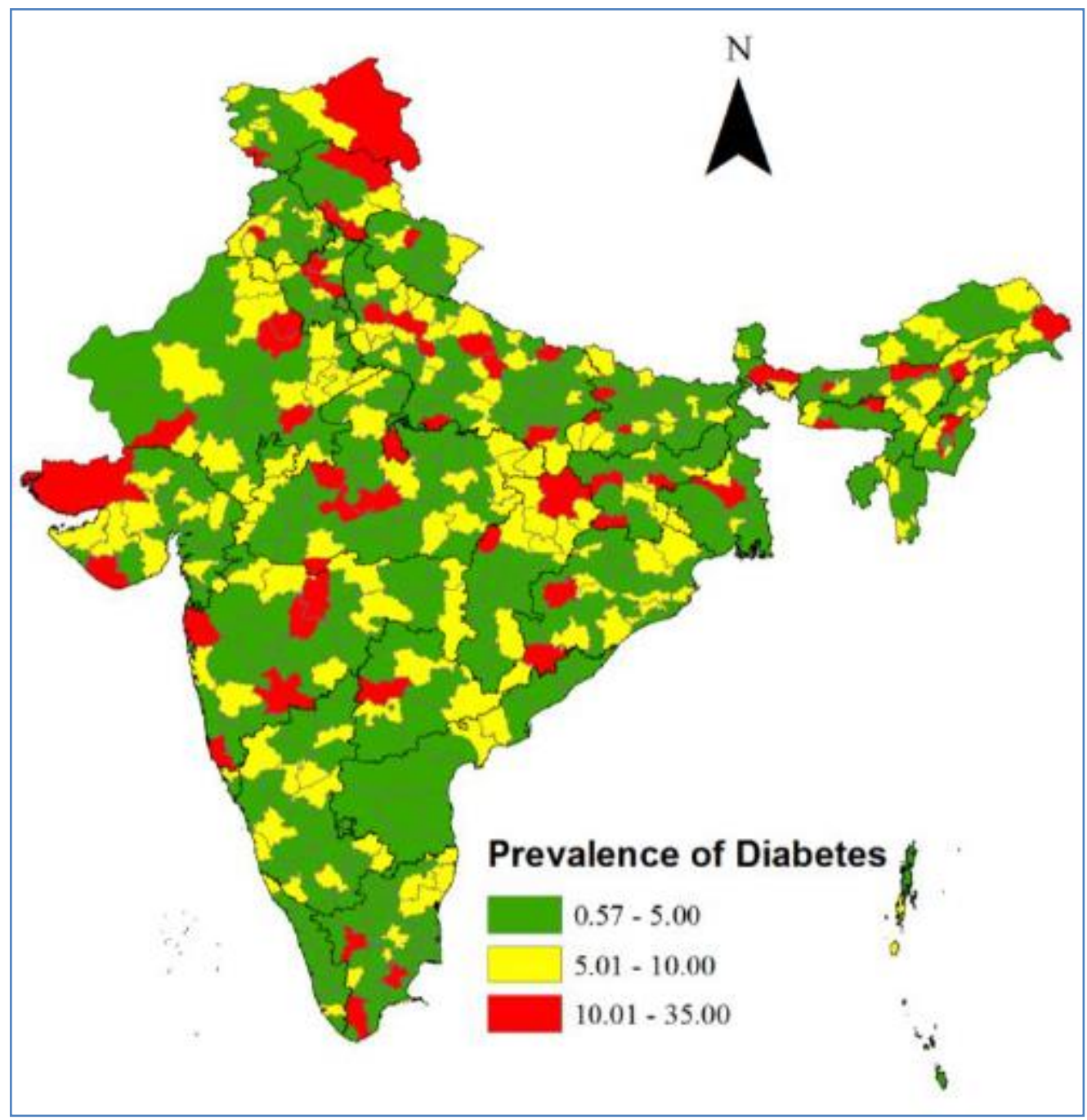

Figure 1: Prevalence of diabetes among older adolescents and young adults across 640 districts of India

\section{Global Moran's I for Prevalence of DM in India}

The results of the study are entirely based on aggregated district-level data. Overall Global Moran's I spatial autocorrelation is 0.61 (p-value $<0.001$; 999 permutations) indicating high spatial autocorrelation and significant positive association with the levels of prevalence of DM between the districts of India. Therefore, it is necessary to analyze spatial clustering at the local level to identify the areas with significant clustering of the prevalence of DM.

\section{LISA Maps for Prevalence of DM in India}

For obtaining the significant clustering of the prevalence of DM, we generate the LISA cluster map. Figure 2 presents the LISA map for the prevalence of DM among older adolescents and young adults. There is significant high-high clustering of the prevalence of DM in the districts of northeastern states, eastern states, southern states and Delhi National Capital Regions (NCR). And, significant lowlow clustering of the prevalence of DM in districts of northern states and central India for which the health care and delivery system are relatively poor. 


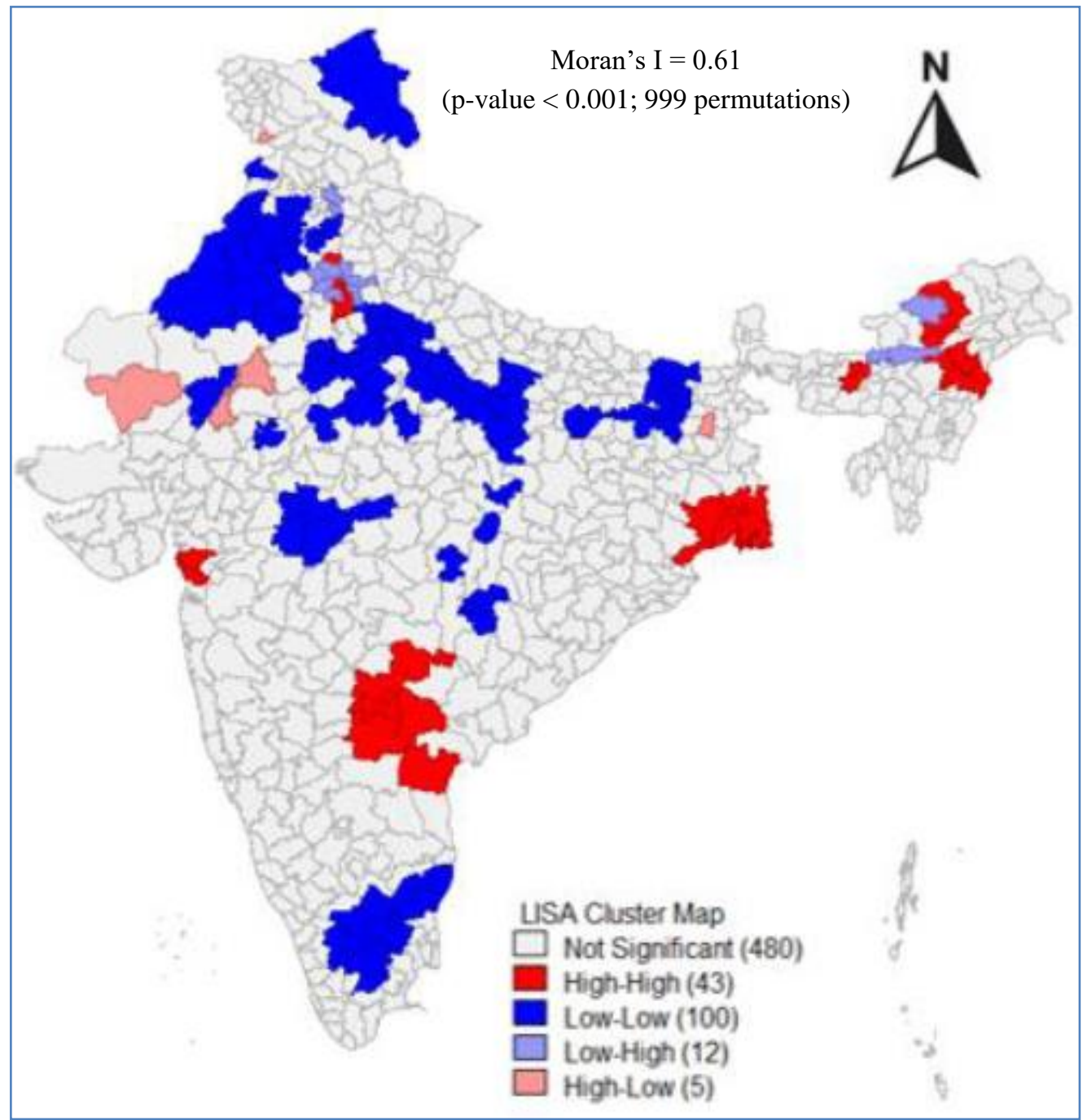

Figure 2: LISA map for the prevalence of DM among older adolescents and young adults

\section{Spatial Regression - Simultaneous Autoregressive Model}

After confirming the presence of significant spatial autocorrelation in the prevalence of DM across 640 districts of India. The spatial effect has been model by using Simultaneous Autoregressive Model (SAR). Table 1 presents the estimates from the SAR model assessing the determinants of DM among older adolescents and young adults in India. The study found the value of lambda $(\lambda)$ capturing spatial effect is positive and highly statistically significant. This indicates the substantial spatial dependence in the prevalence of diabetes across the neighbourhood districts.

Prevalence of DM is likely to increase by 0.040 times as the percentage of urban household increases. Similarly, an increase in the percentage of the overweight population is likely to increase the prevalence of DM by 0.180 times. Behavioural risk factors also have an impact on the prevalence of DM. As the prevalence of tobacco consumption, smoking and drinking of alcohol increases, the prevalence of $\mathrm{DM}$ is likely to increase by $0.123,0.101$, and 0.106 times respectively. The professionals who are physically inactive are also at a higher risk of having DM. As the percentage of professionals who are physically inactive increases, the prevalence of DM is likely to increase by 0.065 times. Compared to districts with a lesser percentage of household with richest wealth quintile 
and median years of education, the prevalence of DM is likely to decrease as the percentage of household with richest wealth quintile and median years of education increases. Prevalence of DM is likely to decrease by 0.051 times if the percentage of household with the richest wealth quintile increases. And the prevalence of DM is likely to decrease by 0.084 times if the median years of the education of the population increases.

Table 1: Estimates from the simultaneous autoregressive model (SAR) assessing determinants of DM among older adolescents and young adults in India.

\begin{tabular}{lc}
\hline Characteristics of Interest & Estimated Coeff. \\
\hline Intercept & 4.241 \\
$\%$ of Urban Household & $0.040^{* * *}$ \\
$\%$ of Overweight Population & $0.189 * * *$ \\
$\%$ of Household in Highest Wealth Quintile & $-0.051^{* * *}$ \\
$\%$ of Household Consuming Veg. \& N-Veg. Occasionally & 0.680 \\
$\%$ of Household Consuming at least One Veg. Daily & 0.655 \\
$\%$ of Household Consuming at least One N-Veg. Daily & 0.659 \\
$\%$ of Household Consuming at least One Veg. \& N-Veg. Daily & 0.702 \\
$\%$ of Household Consuming Fried Food Daily & 0.010 \\
Prevalence of Tobacco Consumption & $0.123 * *$ \\
Prevalence of Smoking & $0.101 * *$ \\
Prevalence of Drinking & $0.106^{* *}$ \\
$\%$ of Professional Who are Physically Inactive & $0.065^{* *}$ \\
Median Years of Education of the population & -0.084 \\
\hline Lambda ( $\lambda)$ & $0.620^{* * *}$ \\
Akaike Information Criteria (AIC) & 3181.8 \\
\hline Note: ** p-value < 0.05 \& *** p-value < 0.01; Veg.- Vegetarian \& N-Veg. - Non-Vegetarian
\end{tabular}

\section{Discussion}

The prevalence of DM is increasing in India and is shifting towards the lower ages (Ramachandran et al. 2001; Wild et al. 2004; Joshi et al. 2007; IDF 2015). The data from the fourth round of National Family Health Survey reveals the prevalence of DM among older adolescents and young adults greater than 10 percent in as many as 59 districts spreading across more than 20 Indian states. In the past decades, there has been evidence of disproportionate distribution of prevalence of DM across the Indian states (Anjana et al. 2017; Arora et al. 2010). Compared to districts in other states, the districts in northern, western, central and northeastern states have a higher prevalence of DM among older adolescents and young adults. The prevalence of DM is highly influenced by the regional variation between the districts. The study indicates the substantial spatial dependence (Moran's I = 0.61) in the prevalence of DM between the 640 districts of Indian states. The prevalence of DM among older adolescents and young adults is high and significantly clustered in the districts of northeastern states, eastern states, southern states and Delhi National Capital Regions (NCR). While the prevalence of DM among older adolescents and young adults is low and significantly clustered in districts of northern states and central India which are typically characterized by poor health indicators accompanied with poor health care and delivery system (Kumar and Prakash 2011; Kumar and Mishra 2015). Compared to the burden of non-communicable diseases, the burden of communicable 
diseases is relatively higher in these regions (Reddy et al. 2005; Upadhyay 2012). Many studies have highlighted the socioeconomic burden of non-communicable diseases particularly DM in India (Bjork et al. 2003; Kapur 2007; Shashank et al. 2008). The study also reveals that as the percentage of the household with the richest wealth quintile and the median years of education increases, the prevalence of DM among older adolescents and young adults is more likely to decreases. But, the impact of urbanization and change in the lifestyle plays a very significant role in increasing the prevalence of DM (Tuomilehto et al. 2001; Whittemore et al. 2002; Simpson et al. 2003; Kosaka et al. 2005; Lindstrom et al. 2006; Rajeski et al. 2012). The prevalence of DM among older adolescents and young adults in districts of Indian states are likely to increases with the increase in the percentage of urban households. Similarly, as the percentage of overweight and professionals who are physically inactive increases, there is higher risk of increasing the prevalence of DM among older adolescents and young adults. The importance of behavioural risk factors in escalating the prevalence of DM cannot be ignored (Rimm et al. 1995; Le et al. 1997; Manson et al. 2000; Shi et al. 2013). As the prevalence of the behavioural risk factors such as tobacco consumption, smoking and drinking of alcohol among older adolescents and young adult's increases, the prevalence of DM is also likely to increase.

\section{Limitations of the study}

Every study is subject to limitations and the study suffers from two inevitable limitations. First, the blood glucose level provided in the survey to define the respondents as diabetic or not is based on the series of biomarkers test and measurements. But, the measurement was taken randomly i.e. whether the respondents were fasting on not prior to the test was not considered. And second, the National Family Health Survey is a cross-sectional survey. Thus, the causality of DM cannot be established from the study. And the prevalence of DM or any characteristic might vary greatly across a district.

\section{Conclusion}

The study shows increasing and widespread prevalence of DM among older adolescents and young adults in India. Since this is a disease which cannot be cured, increasing prevalence among this population would lead to a drastic change in social as well as economic conditions of the country. Urbanization and sedentary habits leading to overweight, professions with inactive physical activities and behavioral risk factors such as smoking, consumption of tobacco and drinking of alcohol are the major risk factor escalating the risk of DM among older adolescents and young adults. Therefore, reduction in the prevalence of DM among this population is necessary and would be possible only if area-specific measures would be adopted in those clusters of districts where DM is high considering the other intervening covariates.

Ethical Statement: The study used secondary data which is available in the public domain. Therefore, the author has no ethical statement to disclose.

Conflict of Interest: The author has no conflict of interest relevant to this article to disclose.

Acknowledgement: The author acknowledges the opportunity provided to present this paper for the International Conference on Public Health and Well Being 2019. 


\section{References}

Anjana, R.M., Deepa, M., Pradeepa, R., Mahanta, J., Narain, K., Das, H.K., Adhikari, P., Rao, P.V., Saboo, B., Kumar, A. and Bhansali, A., 2017. Prevalence of diabetes and prediabetes in 15 states of India: results from the ICMR-INDIAB population-based cross-sectional study. The Lancet Diabetes \& Endocrinology, 5(8), pp.585-596.

Arora, V., Malik, J.S., Khanna, P., Goyal, N., Kumar, N. and Singh, M., 2010. Prevalence of diabetes in urban Haryana. Australasian Medical Journal (Online), 3(8), p.488.

Bjork, S., Kapur, A., King, H., Nair, J. and Ramachandran, A., 2003. Global policy: aspects of diabetes in India. Health Policy, 66(1), pp.61-72.

Bleyer, W.A., 2002. Cancer in older adolescents and young adults: epidemiology, diagnosis, treatment, survival, and importance of clinical trials. Medical and Pediatric Oncology: The Official Journal of SIOP - International Society of Pediatric Oncology (Societé Internationale d'Oncologie Pédiatrique, 38(1), pp.1-10.

Chan, J.M., Rimm, E.B., Colditz, G.A., Stampfer, M.J. and Willett, W.C., 1994. Obesity, fat distribution, and weight gain as risk factors for clinical diabetes in men. Diabetes Care, 17(9), pp.961-969.

Diamond, J., 2011. Medicine: diabetes in India. Nature, 469(7331), p.478.

Gupta, A.K., Ladusingh, L. and Borkotoky, K., 2016. Spatial clustering and risk factors of infant mortality: district-level assessment of high-focus states in India. Genus, 72(1), p.2.

Gupta, R., 2006. Smoking, educational status \& health inequity in India. Indian Journal of Medical Research, 124(1), p.15.

International Diabetes Federation (2015) International Diabetes Federation Diabetes Atlas, 7th edition https://www.idf.org/e-library/epidemiology-research/diabetes-atlas/13-diabetes-atlas-seventh-edition.html

International Institute for Population Sciences (IIPS) and ICF. 2017. National Family Health Survey (NFHS-4), 2015-16: India. Mumbai: IIPS.

Joshi, S.R. and Parikh, R.M., 2007. India; the diabetes capital of the world: Now heading towards hypertension. Journal-Association of Physicians of India, 55(Y), p.323.

Kapur, A., 2007. Economic analysis of diabetes care. Indian Journal of Medical Research, 125(3), p.473.

Koch, M., Gradaus, F., Schoebel, F.C., Leschke, M. and Grabensee, B., 1997. Relevance of conventional cardiovascular risk factors for the prediction of coronary artery disease in diabetic patients on renal replacement therapy. Nephrology, dialysis, transplantation: official publication of the European Dialysis and Transplant Association-European Renal Association, 12(6), pp.1187-1191.

Kosaka, K., Noda, M. and Kuzuya, T., 2005. Prevention of type 2 diabetes by lifestyle intervention: a Japanese trial in IGT males. Diabetes Research and Clinical Practice, 67(2), pp.152-162.

Kumar, C. and Prakash, R., 2011. Public-private dichotomy in utilization of health care services in India. Consilience, (5), pp.25-52.

Kumar, V. and Mishra, A.J., 2015. Quality of health care in primary health care system: a reflection from Indian state. International Journal of Health System and Disaster Management, 3(3), p.136.

Le Marchand, L., Wilkens, L.R., Kolonel, L.N., Hankin, J.H. and Lyu, L.C., 1997. Associations of sedentary lifestyle, obesity, smoking, alcohol use, and diabetes with the risk of colorectal cancer. Cancer Research, 57(21), pp.4787-4794.

Lindström, J., Ilanne-Parikka, P., Peltonen, M., Aunola, S., Eriksson, J.G., Hemiö, K., Hämäläinen, H., Härkönen, P., Keinänen-Kiukaanniemi, S., Laakso, M. and Louheranta, A., 2006. Sustained reduction in the incidence of type 2 diabetes by lifestyle intervention: follow-up of the Finnish Diabetes Prevention Study. The Lancet, 368(9548), pp.1673-1679.

Manson, J.E., Ajani, U.A., Liu, S., Nathan, D.M. and Hennekens, C.H., 2000. A prospective study of cigarette smoking and the incidence of diabetes mellitus among US male physicians. The American Journal of Medicine, 109(7), pp.538-542. 
Martín-Timón, I., Sevillano-Collantes, C., Segura-Galindo, A. and del Cañizo-Gómez, F.J., 2014. Type 2 diabetes and cardiovascular disease: have all risk factors the same strength?. World Journal of Diabetes, 5(4), p.444.

Mohan, V., Anjana, R.M., Pradeepa, R., Unnikrishnan, R., Tanvir, K. and Das, A.K., 2017. The ICMR INDIAB Study-A Compendium of Type 2 Diabetes in India: Lessons Learnt for the Nation. API.

Mohan, V., Mathur, P., Deepa, R., Deepa, M., Shukla, D.K., Menon, G.R., Anand, K., Desai, N.G., Joshi, P.P., Mahanta, J. and Thankappan, K.R., 2008. Urban rural differences in prevalence of self-reported diabetes in India-The WHO-ICMR Indian NCD risk factor surveillance. Diabetes Research and Clinical Practice, 80(1), pp.159-168.

Mokdad, A.H., Ford, E.S., Bowman, B.A., Dietz, W.H., Vinicor, F., Bales, V.S. and Marks, J.S., 2003. Prevalence of obesity, diabetes, and obesity-related health risk factors, 2001. Journal of American Medical Association, 289(1), pp.76-79.

Mote, B.N., 2016. A regional epidemiology of India's "NCD's risk factors" focusing particularly on Maharashtra: A call for "Health promotion" once again. International Journal of Medicine and Public Health, 6(1).

Pereira, M.A., Kartashov, A.I., Ebbeling, C.B., Van Horn, L., Slattery, M.L., Jacobs Jr, D.R. and Ludwig, D.S., 2005. Fast-food habits, weight gain, and insulin resistance (the CARDIA study): 15-year prospective analysis. The Lancet, 365(9453), pp.36-42.

Ramachandran, A., Snehalatha, C., Kapur, A., Vijay, V., Mohan, V., Das, A.K., Rao, P.V., Yajnik, C.S., Kumar, K.P., Nair, J.D. and Diabetes Epidemiology Study Group in India (DESI, 2001. High prevalence of diabetes and impaired glucose tolerance in India: National Urban Diabetes Survey. Diabetologia, 44(9), pp.1094-1101.

Ramachandran, A., Snehalatha, C., Latha, E., Manoharan, M. and Vijay, V., 1999. Impacts of urbanisation on the lifestyle and on the prevalence of diabetes in native Asian Indian population. Diabetes Research and Clinical Practice, 44(3), pp.207-213.

Reddy, K.S., Shah, B., Varghese, C. and Ramadoss, A., 2005. Responding to the threat of chronic diseases in India. The Lancet, 366(9498), pp.1744-1749.

Rejeski, W.J., Ip, E.H., Bertoni, A.G., Bray, G.A., Evans, G., Gregg, E.W. and Zhang, Q., 2012. Lifestyle change and mobility in obese adults with type 2 diabetes. New England Journal of Medicine, 366(13), pp.1209-1217.

Rimm, E.B., Chan, J., Stampfer, M.J., Colditz, G.A. and Willett, W.C., 1995. Prospective study of cigarette smoking, alcohol use, and the risk of diabetes in men. BMJ, 310(6979), pp.555-559.

Robert Gentleman, Kurt Hornik, Giovanni Parmigiani (2008). Applied Spatial Data Analysis with R.

Savoca, M.R., Miller, C.K. and Ludwig, D.A., 2004. Food habits are related to glycemic control among people with type 2 diabetes mellitus. Journal of the American Dietetic Association, 104(4), pp.560-566.

Shashank, R.J., Das, A.K., Vijay, V.J. and Mohan, V., 2008. Challenges in diabetes care in India: sheer numbers, lack of awareness and inadequate control. Journal of Association of Physicians of India, 56(6), pp.443-450.

Shi, L., Shu, X.O., Li, H., Cai, H., Liu, Q., Zheng, W., Xiang, Y.B. and Villegas, R., 2013. Physical activity, smoking, and alcohol consumption in association with incidence of type 2 diabetes among middle-aged and elderly Chinese men. PloS One, 8(11), p.e77919.

Simpson, R.W., Shaw, J.E. and Zimmet, P.Z., 2003. The prevention of type 2 diabetes - lifestyle change or pharmacotherapy? A challenge for the 21st century. Diabetes Research and Clinical Practice, 59(3), pp.165-180.

Sowers, J.R., Levy, J. and Zemel, M.B., 1988. Hypertension and diabetes. Medical Clinics of North America, 72(6), pp.1399-1414.

Steppan, C.M., Bailey, S.T., Bhat, S., Brown, E.J., Banerjee, R.R., Wright, C.M., Patel, H.R., Ahima, R.S. and Lazar, M.A., 2001. The hormone resistin links obesity to diabetes. Nature, 409(6818), p.307.

Tuomilehto, J., Lindström, J., Eriksson, J.G., Valle, T.T., Hämäläinen, H., Ilanne-Parikka, P., KeinänenKiukaanniemi, S., Laakso, M., Louheranta, A., Rastas, M. and Salminen, V., 2001. Prevention of type 2 
diabetes mellitus by changes in lifestyle among subjects with impaired glucose tolerance. New England Journal of Medicine, 344(18), pp.1343-1350.

Tuttolomondo, A., Maida, C. and Pinto, A., 2015. Diabetic foot syndrome as a possible cardiovascular marker in diabetic patients. Journal of Diabetes Research, 2015.

Upadhyay, R.P., 2012. An overview of the burden of non-communicable diseases in India. Iranian Journal of Public Health, 41(3), p.1.

Whittemore, R., Chase, S.K., Mandle, C.L. and Roy, C., 2002. Lifestyle change in type 2 diabetes: A process model. Nursing Research, 51(1), pp.18-25.

Wild, S., Roglic, G., Green, A., Sicree, R. and King, H., 2004. Global prevalence of diabetes: estimates for the year 2000 and projections for 2030. Diabetes Care, 27(5), pp.1047-1053. 